\title{
Campo Volcánico Monogenético Villamaría-Termales, Cordillera Central, Andes colombianos (Parte I): Características morfológicas y relaciones temporales
}

\author{
Luis Alvaro Botero-Gómez ${ }^{1,4 *}$; Pablo Osorio²; Hugo Murcia ${ }^{3,4}$; Carlos Borrero; Jeny Alejandra Grajales ${ }^{5}$
}

DOI: http://dx.doi.org/10.18273/revbol.v40n3-2018005@@

Forma de citar: Botero-Gómez, L.A., Osorio, P., Murcia, H., Borrero, C., y Grajales, J.A. (2018). Campo Volcánico Monogenético Villamaría-Termales, Cordillera Central, Andes colombianos (Parte I): Características morfológicas y relaciones temporales. Boletín de Geología, 40(3), 85-102. DOI: 10.18273/revbol.v40n3-2018005.

\section{RESUMEN}

El Campo Volcánico Monogenético Villamaría-Termales (CVMVT) está localizado en la parte media de la Cordillera Central de Colombia y es parte de la Provincia Volcano Tectónica San Diego - Cerro Machín (PVTSC). Este campo está relacionado con el Complejo Volcánico Nevado del Ruiz (CVNR) y está compuesto de al menos 14 volcanes monogenéticos, de los cuales 12 centros volcánicos son cónicos y subcónicos (domos), uno macizo (domo), además de un irregular (flujo de lava). A partir de análisis morfométricos y geomorfológicos, se define el CVMVT como un campo volcánico que ocupa un área de $114,5 \mathrm{~km}^{2}$, afectado por diferentes procesos geomorfológicos y glaciales. Estos centros volcánicos tienen un rango de edad de 1,8 Ma a <38.000 años (Calabriense--Pleistoceno Tardío) y una relación con el sistema de fallas Villamaría-Termales. Los análisis morfométricos como volumen e índices de separación y agrupamiento permitieron definir la relación espacial (i.e. distancia entre los volcanes y sus geoformas) de los centros volcánicos que conforman el CVMVT.

Palabras clave: Morfometría; domo de lava; flujo de lava; vulcanismo monogenético; Andes colombianos.

\section{The Villamaría-Termales Monogenetic Volcanic Field, Central Cordillera, colombian Andes (Part I): Morphological features and temporal relationships}

\begin{abstract}
The Villamaría-Termales Monogenetic Volcanic Field (VTMVF) is located in the middle of the Central Cordillera of Colombia. It is part of the San Diego - Cerro Machín Volcano Tectonic Province (SCVTP), and is related to the Nevado del Ruiz Volcanic Complex (NRVC). The VTMVF is composed of at least 14 monogenetic volcanoes; 12 of them are conical and subconical (domes), one is massif (dome), and one is irregular (lava flow). Based on the morphometric and geomorphological analysis reported in this study, we define the VTMVF as a volcanic field affected by a variety of geomorphological and glacial processes. This field occupies an area of $114.5 \mathrm{~km}^{2}$, where the Villamaría-Termales fault system controlled the volcanic dispersion. These volcanic centers have an age range between 1.8 Ma and $<38,000$ years (Calabrian-Late Pleistocene). The morphometric analysis as volume and separation and grouping indexes allowed us to define the spatial relationship (i.e. distance between the volcanoes and their geoforms) between the volcanic centers that make up the VTMVF.
\end{abstract}

Keywords: Morphometry; lava dome; lava flow; monogenetic volcanism; colombian Andes.

\footnotetext{
${ }^{1}$ Maestría en Ciencias de la Tierra, Universidad de Caldas, Manizales, Colombia. (*) lualbogo1993@hotmail.com

${ }^{2}$ Programa de Geología, Universidad de Caldas, Manizales, Colombia. pablosov@outlook.es

${ }^{3}$ Departamento de Ciencias Geológicas, Universidad de Caldas, Manizales, Colombia. hugo.murcia@ucaldas.edu.co

${ }^{4}$ Instituto de Investigación en Estratigrafía, Universidad de Caldas Manizales, Colombia. borrero_c@yahoo.com

${ }^{5}$ Universidad de Concepción, Concepción, Chile. jgrajales@udec.cl
} 


\section{INTRODUCCIÓN}

Un campo volcánico sensu stricto es un término utilizado para agrupar múltiples centros volcánicos, las cuales se encuentran en un área específica (e.g. Connor y Conway, 2000; Németh, 2010; Le Corvec et al., 2013). Estos volcanes pueden ser domos de lava, flujos de lava, conos piroclásticos, conos de toba, anillos de toba y maares (Kereszturi y Németh, 2012; De Silva y Lindsay, 2015; Miallier et al., 2017; Shane y Coote, 2018). Así, los campos volcánicos deben ser definidos por factores como: génesis de los centros volcánicos (i.e. igual fuente de magma?), distancia entre los focos de erupción, relaciones composicionales de los productos de la erupción e interacción en profundidad de los edificios volcánicos (Cañón-Tapia, 2016). Aunque la cantidad de volcanes es un valor relativo, trabajos como los de Connor y Conway (2000) y Le Corvec et al. (2013) han propuesto valores mayores a 50 y 10 edificios volcánicos, respectivamente para delimitar un campo volcánico. En Colombia, campos volcánicos monogenéticos han sido definidos con menos de 10 volcanes (Murcia et al., en prensa).

En Colombia, en el flanco W de la Cordillera Central, al NW del Complejo Volcánico Nevado del Ruiz (CVNR; Martínez et al., 2014) (FIGURA 1), existen al menos 14 centros volcánicos monogenéticos, los cuales forman entre ellos un campo volcánico que se extiende hasta 30 $\mathrm{km}$ del complejo. Éstos son denominados formalmente

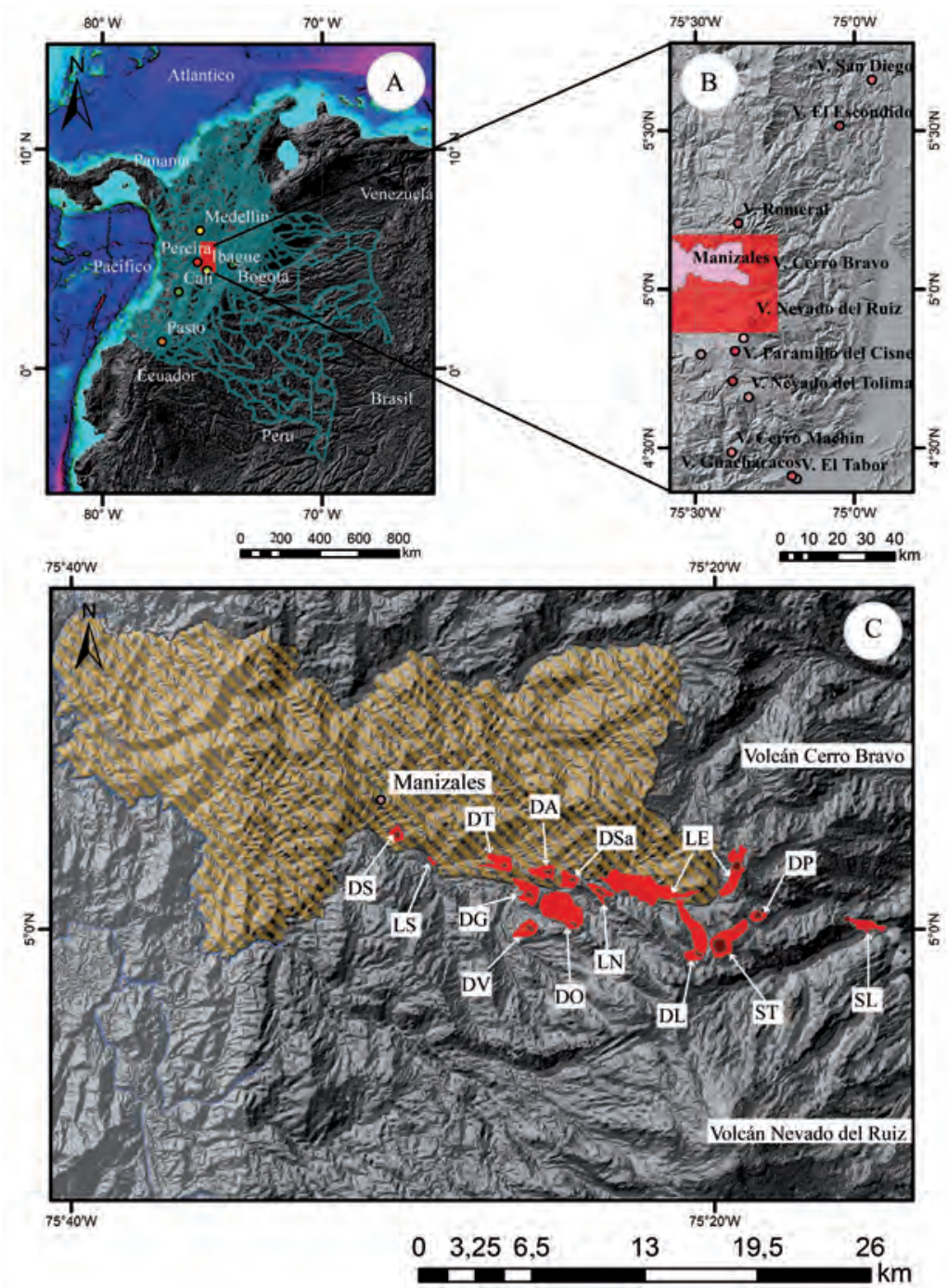

FIGURA 1. A. Localización de la Provincia Volcano Tectónica San Diego - Cerro Machín (PVTSC) en la Cordillera Central de Colombia (rectángulo rojo). B. Ubicación de la ciudad de Manizales, el CVMVT y el CVNR dentro de la PVTSC. C. Ubicación de los domos monogenéticos del CVMVT respecto a la ciudad de Manizales; como referencia los volcanes Cerro Bravo y Nevado del Ruiz. DS: Domo Sancancio, LS: Flujo de lava de Lusitania, DT: Domo Tesorito, DG: Domo Gallinazo, DV: Domo Victoria, DA: Domo Amazonas, DO: Domo La Oliva, DSa: Domo Sabinas, LN: Domo La Negra, LE: Domo La Esperanza, DL: Domo La Laguna, ST: Domo Santana, DP: Domo El Plato y SL: Domo San Luis. 
en este trabajo como Campo Volcánico Monogenético Villamaría-Termales (CVMVT) y corresponden a los siguientes volcanes: domos Sancancio, Tesorito, Gallinazo, Amazonas, Victoria, Sabinas, La Oliva, La Negra, La Esperanza, La Laguna, Santana, El Plato y San Luis, y Flujo de lava de Lusitania (Naranjo y Ríos, 1989; Thouret et al., 1990; Ancochea et al., 1991; Aristizabal y Echeverry, 2001; Montoya y Torres, 2005; Toro et al., 2008; Ayala, 2009; Borrero et al., 2009; Mejía et al., 2011; Rayo, 2012; Martínez et al., 2014; FIGURA 1C).

Este estudio presenta un análisis morfométrico, geomorfológico y estructural de cada uno de los volcanes que caracterizan el CVMVT. Adicionalmente se presentan las relaciones espacio-temporales con base en menciones previas de la existencia de domos y flujos de lava en el sector (FIGURA 1). Finalmente, se relaciona el CVMVT con el ambiente tectónico, el vulcanismo cercano y en general el magmatismo asociado en la región.

\section{GEOLOGÍA Y VULCANISMO}

El CVMVT se encuentra localizado en los municipios de Manizales y Villamaría en el departamento de Caldas, en el flanco W de la Cordillera Central de Colombia. Éste se ubica en el sector centro de la Provincia Volcano Tectónica San Diego - Cerro Machín (PVTSC; Martínez et al., 2014) (FIGURA 1B), al NW del Complejo Volcánico Nevado del Ruiz y al SW del volcán Cerro Bravo (FIGURA 1C). El basamento del campo volcánico, está constituido por rocas metamórficas Triásicas del Complejo Cajamarca, rocas volcanoclásticas Cretácicas del Complejo Quebradagrande, rocas miloníticas Cretácicas del Guacaica y unidades ígneas Paleógenas del Stock de Manizales y del Batolito de El Bosque (Cuellar et al., 2003; Villagómez et al., 2011; Villagómez y Spikings, 2013; Gómez et al., 2015; González-Garcia y Jessell, 2016; FIGURA 2).

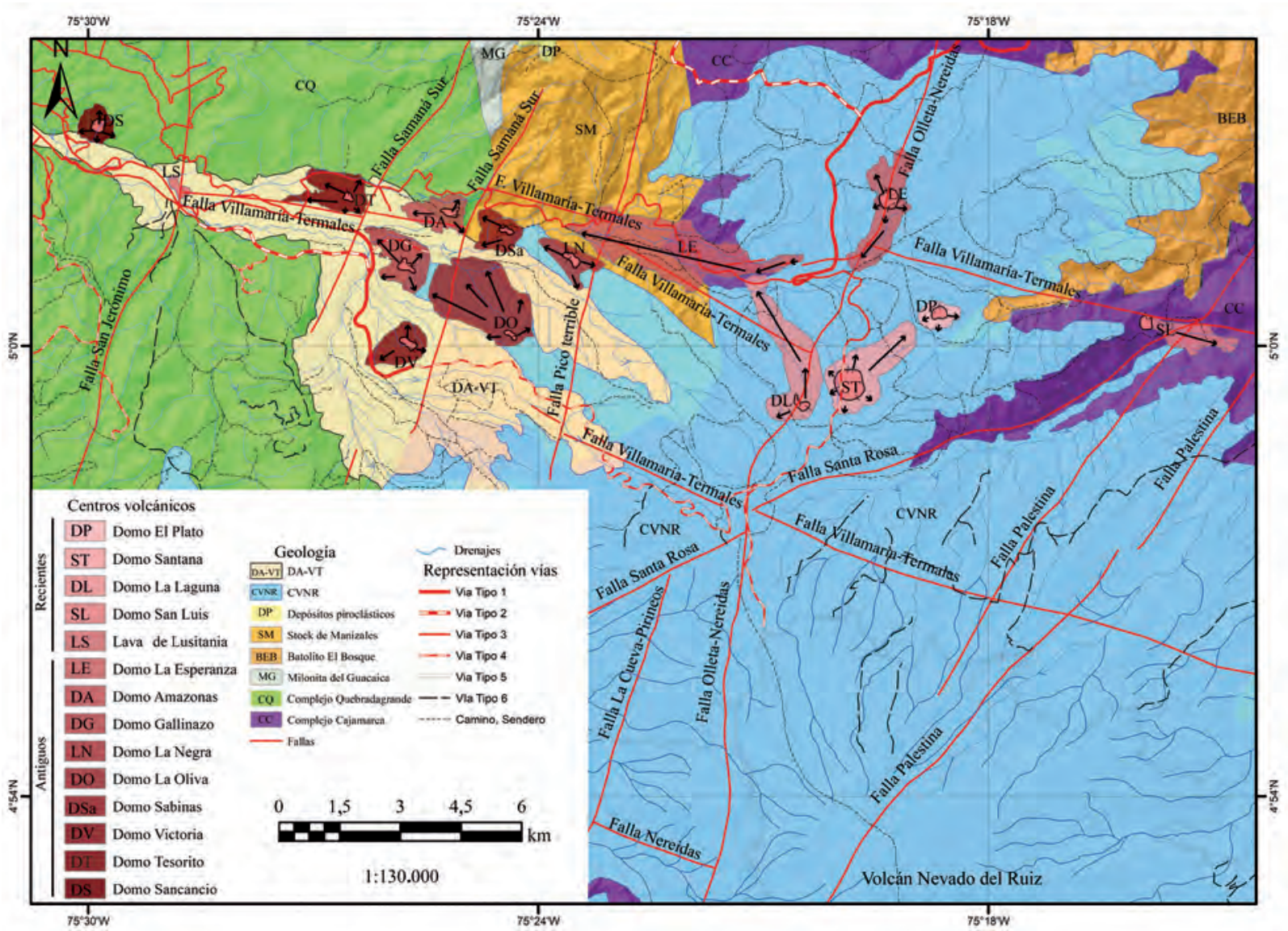

FIGURA 2. Mapa geológico del Campo Volcánico Monogenético Villamaría-Termales (CVMVT). Las expresiones monogenéticas representadas con tonalidades rojas según sus rangos de temporalidad. 


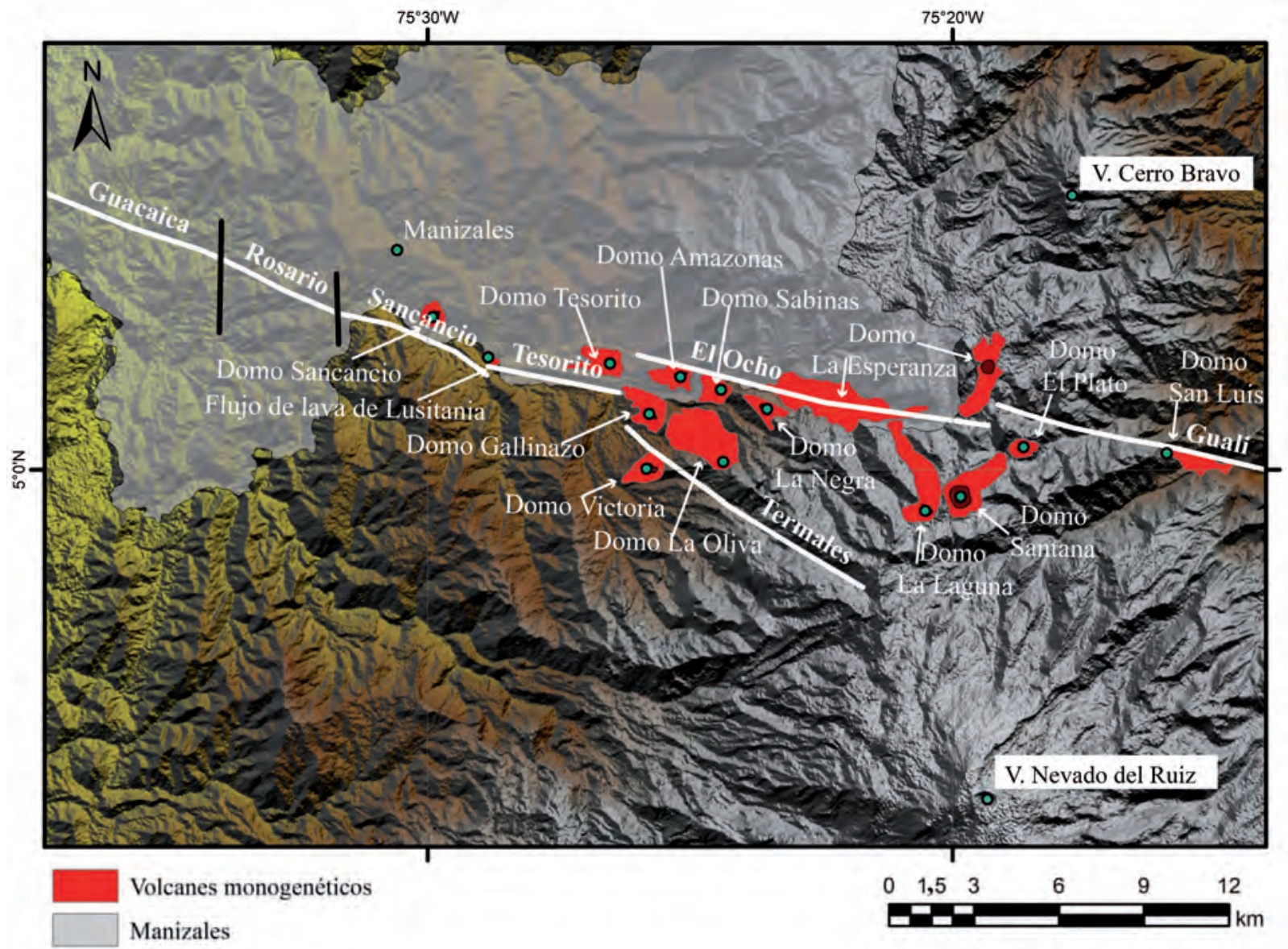

FIGURA 3. Segmentos del sistema de fallas Villamaría-Termales, y domos del Campo Volcánico Monogenético VillamaríaTermales. Nótese la disposición de los centros volcánicos sobre los trazos del sistema de fallas. Las líneas blancas ilustran los segmentos del SFVT y las negras corresponden a barreras estructurales y no corresponden a fallas geológicas.

Estructuralmente, los volcanes se encuentran asociados al sistema de fallas normal sinistral VillamaríaTermales (SFVT) con dirección predominante N65W (González y Jaramillo, 2002), el cual se encuentra dividido en siete segmentos (FIGURA 3; TABLA 1) limitados por barreras estructurales y lineamientos en dirección N-S y NE-SW.

Según Borrero et al.(2009), los volcanes del campo aquí definido como CVMVT, se encuentran emparentados espacial, estratigráfica y geoquímicamente con los productos del CVNR. De hecho, este conjunto de volcanes ha sido considerado como parte de la evolución del CVNR, la mayoría de ellos dentro del denominado Ruiz Ancestral (Thouret et al., 1990) o periodo eruptivo Pre-Ruiz (Martínez et al., 2014). Para el CVMVT se conocen edades K/Ar de dos de los volcanes (Domo Sancancio o Cerro La Cruz con 1,2 \pm 0,08 Ma, y Domo Tesorito con 1,2 \pm 0,2 Ma; Thouret et al., 1990), y una más que por relaciones estratigráficas $\mathrm{y}$ de paleomagnetismo corresponde $\mathrm{a}<38.000 \pm 16$ años (Flujo de lava de Lusitania; Mejía et al., 2011). Por relaciones estratigráficas, algunos volcanes también se asumen como más jóvenes que $0,045-0,035 \mathrm{Ma}$, teniendo en cuenta que se encuentran suprayacidos por un depósito de avalancha de escombros (Villamaria Termales; DA-VT; Martínez et al., 2014), el cual se asume más reciente que la edad del Máximo Avance Glaciar durante la Última Glaciación definida para esta región de Colombia (cf. Flórez, 1992). 
TABLA 1. Centros volcánicos del CVMVT y la distancia a la que se encuentran de los segmentos del SFVT.

\begin{tabular}{ccc}
\hline Expresión volcánica & Distancia (m) en la horizontal & $\begin{array}{c}\text { Segmento de falla del } \\
\text { SFVT }\end{array}$ \\
\hline Domo Sancancio & 448 & Sancancio \\
Flujo de lava de Lusi- & 0 & Tesorito \\
tania & 524 & Tesorito \\
Domo Tesorito & 194 & Tesorito \\
Domo Gallinazo & 0 & El Ocho \\
Domo Amazonas & 590 & Termales \\
Domo La Oliva & 360 & El Ocho \\
Domo La Negra & 0 & Termales \\
Domo Victoria & 0 & El Ocho \\
Domo Sabinas & 0 & El Ocho \\
Domo La Esperanza & 286 & El Ocho \\
Domo La Laguna & 1048 & El Ocho \\
Domo Santana & 850 & Gualí \\
Domo El Plato & 0 & Gualí \\
Domo San Luis & &
\end{tabular}

\section{METODOLOGÍA}

Para realizar la caracterización morfométrica de los centros volcánicos del CVMVT, se adaptó la metodología propuesta por Samacá-Torres (2016), quien usó ciertos parámetros morfométricos y relaciones espaciales de autores clásicos (Porter, 1972; Settle, 1979; Wood, 1980; Favalli et al., 2009; Grosse et al., 2009; Karátson et al., 2010), para la descripción de volcanes compuestos y conos de escoria monogenéticos en la Cadena Volcánica de los Coconucos (Colombia). En este estudio se aplicó la metodología propuesta para volcanes compuestos ya que se ajusta de manera más adecuada a los domos de lava del CVMVT. Ésta se basa en determinar parámetros como área, volumen, índices de agrupamiento (i.e. distancias de las bases de los edificios volcánicos entre sí), separación (i.e. distancia entre el centro geométrico de un edificio volcánico respecto al más próximo) y elipticidad (relación del eje horizontal más largo que atraviesa el centro volcánico respecto al área del domo). Se realizó una descripción de los domos conforme a la clasificación respecto a la relación entre la altura y el ancho de la base del centro volcánico, desde una perspectiva bidimensional en planta y tridimensional de perfil (Grosse et al., 2009). Asimismo, como la mayoría de los volcanes son generalmente domos de lava con un flujo de lava asociado, se calculó el avance del flujo de lava desde el centro de emisión. Posteriormente, se realizó una comparación de los valores obtenidos para cada centro volcánico.

Para la caracterización espacio-temporal, se determinaron las relaciones cronoestratigráficas con base en observaciones de campo y en el análisis de estudios previos (e.g. Mejía et al., 2011; Martínez et al., 2014); de esta manera se asignó un rango temporal a cada volcán. Adicionalmente, se realizó un análisis geomorfológico a partir de perfiles topográficos de cada centro volcánico, que permitió reconocer la evolución de los edificios volcánicos en función de los procesos de degradación y denudación. Finalmente, se realizó un análisis estructural con base en la literatura existente y de relaciones de campo apoyadas con modelos digitales de elevación del terreno con 12,5 $\mathrm{m}$ de resolución, descargados de la página web de la NASA, usando el satélite ALOS PALSAR y fotografías aéreas del Instituto Geográfico Agustín Codazzi (vuelo C-1145 179-183), usando el sistema de información geográfica Quantum GIS (QGIS).

\section{MORFOMETRÍA Y RELACIONES ESPACIALES}

Los volcanes del CVMVT son, desde una perspectiva bidimensional en planta, dominantemente circulares elongados, con excepción de los domos La Laguna y San Luis que son muy elongados hacia el NNW y 
SEE, respectivamente, y el Flujo de lava de Lusitania que, por sus características intrínsecas de flujo, posee una forma irregular. En general estos volcanes son clasificados tridimensionalmente entre cónicos y subcónicos, excluyendo el Domo La Esperanza, que se clasifica como una estructura maciza (cf. Grosse et al., 2009) (FIGURA 4).

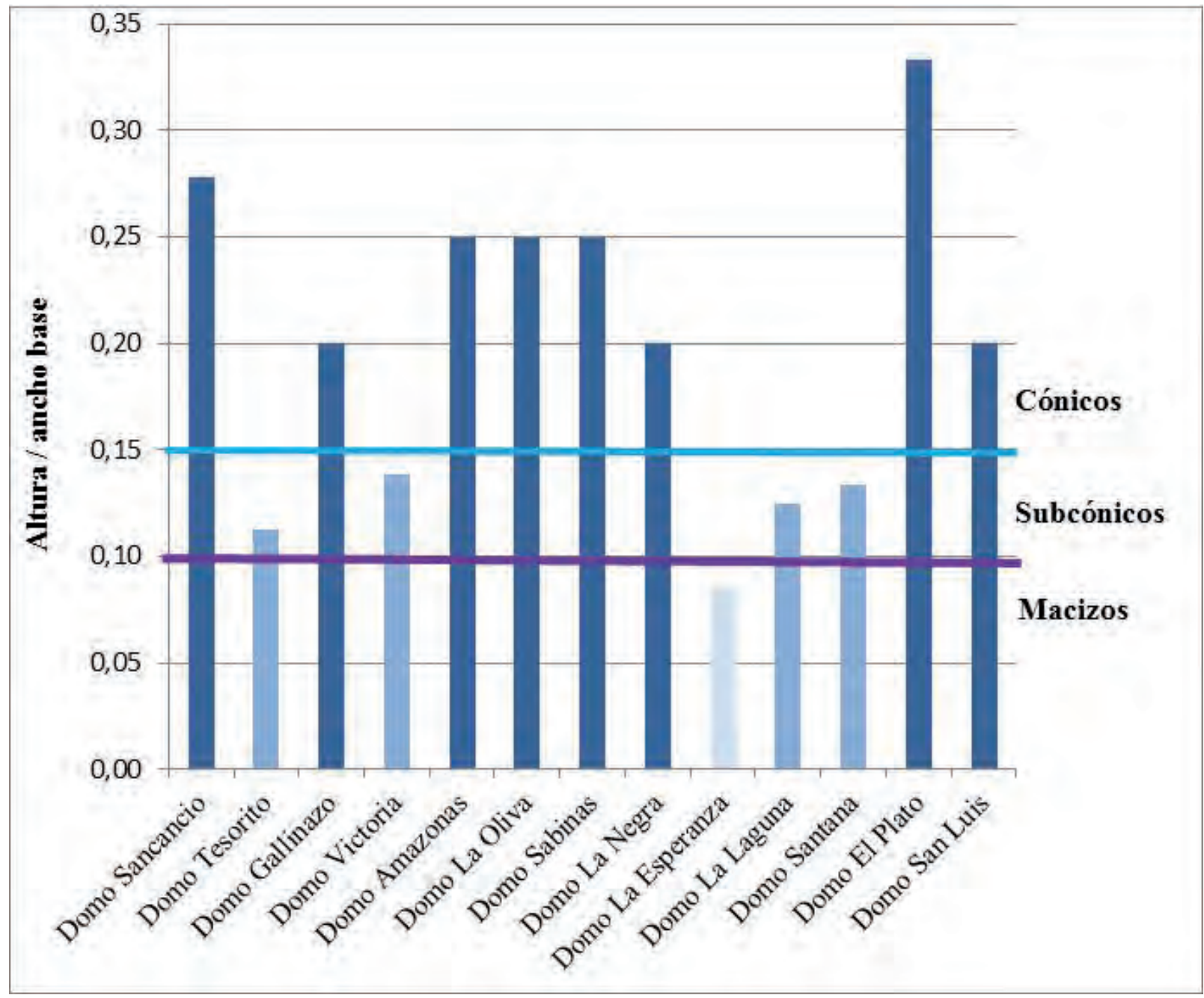

FIGURA 4. Relación (altura / ancho base) de los domos pertenecientes al CVMVT. Valores superiores a 0,15 corresponden a domos cónicos, entre 0,10 - 0,15 subcónicos, y menores a 0,1 como macizos, según Grosse et al. (2009); la diferencia de tonalidad del color azul se realiza según su clasificación. El Flujo de lava de Lusitania no forma parte de este análisis debido a su naturaleza irregular.

El volcán más voluminoso $\left(2,46 \mathrm{~km}^{3}\right)$ del CVMVT es el Domo La Laguna. Las mayores alturas (400 m) corresponden a los domos Gallinazo, Amazonas, La Oliva, El Plato y Sabinas. Los volcanes que ocupan mayor área son La Esperanza $\left(7,33 \mathrm{~km}^{2}\right)$, La Oliva $\left(3,79 \mathrm{~km}^{2}\right)$, La Laguna $\left(2,86 \mathrm{~km}^{2}\right)$, Santana $\left(2,53 \mathrm{~km}^{2}\right)$ y Gallinazo $\left(1,51 \mathrm{~km}^{2}\right)$. El flujo de lava asociado al
Domo La Esperanza es el de mayor avance $(8,88 \mathrm{~km})$, además este flujo de lava presenta una segmentación generada por la erosión, la cual es facilitada por la falla Olleta-Nereidas; seguidos de los flujos de lava asociados a los domos La Laguna, La Oliva, San Luis y Santana (FIGURA 2; TABLA 2). 
Luis Alvaro Botero-Gómez, Pablo Osorio, Hugo Murcia, Carlos Borrero, Jeny Alejandra Grajales

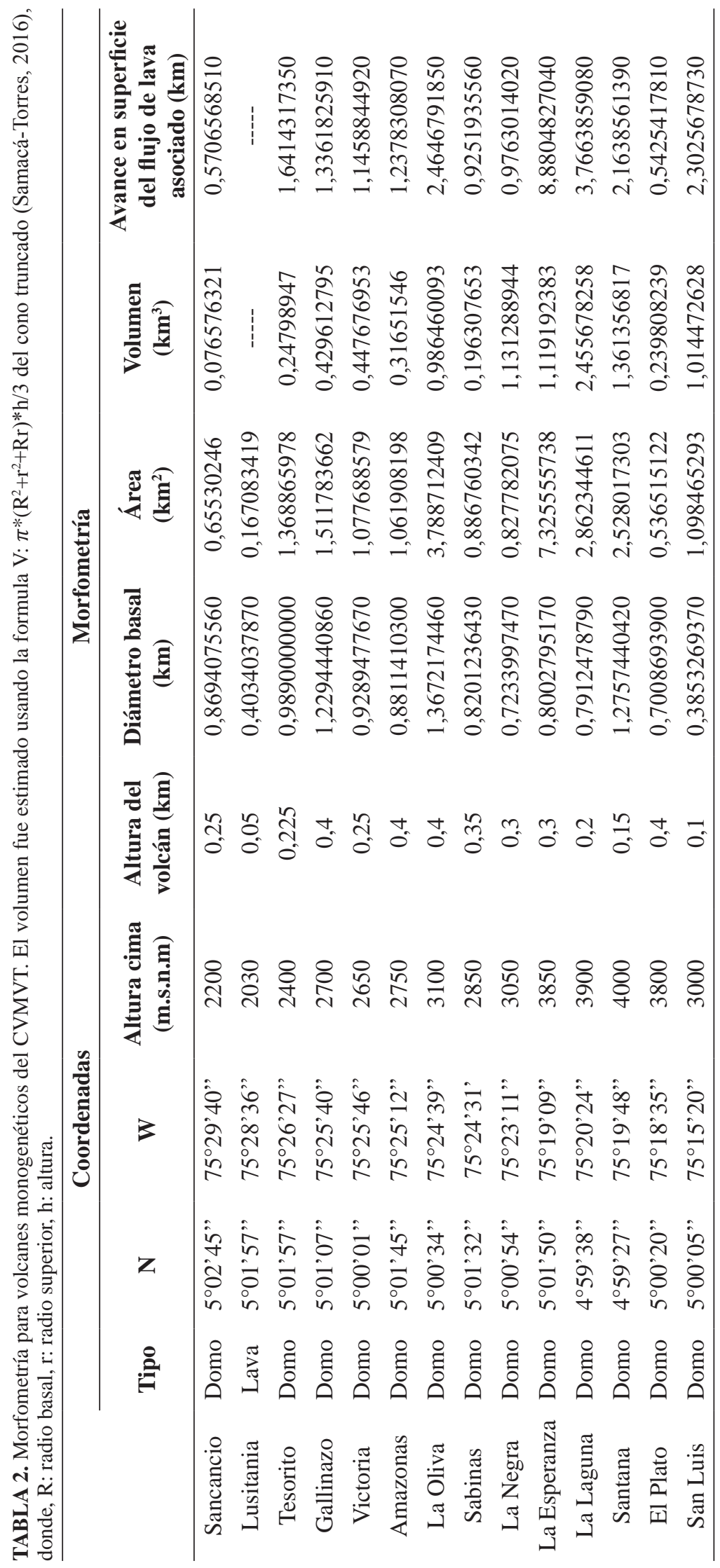


Con respecto a la relación espacial, los índices de agrupamiento (IA) arrojan resultados cercanos a cero (entre 0,035-0,547. i.e. los volcanes se encuentran muy próximos entre sí), con excepción de los centros volcánicos Domo Sancancio, Flujo de lava de Lusitania y Domo San Luis. En cuanto a los índices de separación, los valores son relativamente pequeños (en km) a excepción del Domo San Luis $(5,123$ km), el cual es el volcán monogenético cuyo centro de emisión se encuentra más apartado de algún otro centro volcánico del campo (TABLA 3). Estos índices permiten obtener una aproximación de la dinámica eruptiva, los cuales relacionan un sistema de transporte homogéneo con un comportamiento efusivo (domos y flujos de lava) en este sector.

TABLA 3. Índices de separación, agrupamiento e índice de elipticidad de los volcanes pertenecientes al CVMVT. IE: $\left(\pi *(\text { LongEje } / 2)^{2} /\right.$ Area $)$ de Grosse et al. (2009) donde IE cercanos a 1 es circular, los valores mayores elongados.

\begin{tabular}{ccccc}
\hline Expresión volcánica & IA (km) & IS (km) & $\begin{array}{c}\text { Índice de elipticidad } \\
\text { (IE) }\end{array}$ & $\begin{array}{c}\text { Clasificación (Grosse et al., } \\
\text { 2009) }\end{array}$ \\
\hline Domo Sancancio & 1,593 & 2,46 & 0,9708075 & Circular \\
Flujo de lava de Lusitania & 1,593 & ---- & ---- & Irregular \\
Domo Tesorito & 0,335 & 2,334 & 2,295033 & Circular elongado \\
Domo Gallinazo & 0,125 & 1,453 & 2,0780703 & Circular elongado \\
Domo Victoria & 0,547 & 1,856 & 2,361248 & Circular elongado \\
Domo Amazonas & 0,304 & 1,422 & 1,8934022 & Circular elongado \\
Domo La Oliva & 0,125 & 2,323 & 1,1940451 & Circular \\
Domo Sabinas & 0,218 & 1,422 & 1,7359599 & Circular elongado \\
Domo La Negra & 0,089 & 1,91 & 1,7721028 & Circular elongado \\
Domo La Esperanza & 0,035 & 3,07 & 1,3133649 & Circular elongado \\
Domo La Laguna & 0,044 & 1,3 & 4,3902368 & Muy elongado \\
Domo Santana & 0,044 & 1,3 & 2,7960977 & Circular elongado \\
Domo El Plato & 0,144 & 2,7 & 2,107999 & Circular elongado \\
Domo San Luis & 4,418 & 5,123 & 4,4687243 & Muy elongado \\
\hline
\end{tabular}

El Domo La Esperanza y su flujo de lava asociado (FIGURA 2), es el volcán con mayor envergadura (i.e. la mayor distancia de los extremos de las bases de los domos o diámetro mayor) respecto a su avance y conservación actual, mientras que el Flujo de lava de Lusitania es el centro volcánico de menor extensión.
El Domo Gallinazo (FIGURA 5A) es el de menor diámetro superior presentando un pico que sobresale. El Domo Victoria (FIGURA 5A) es el de mayor diámetro superior, con una superficie plana característica y una disección en medio del domo. 
(A)

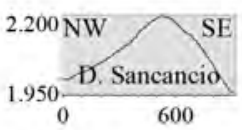

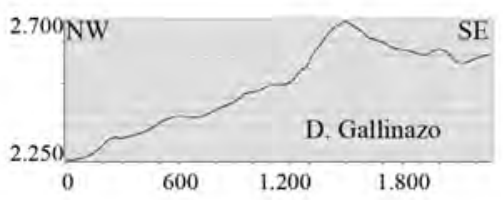

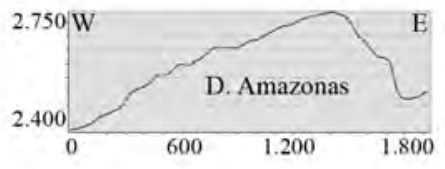

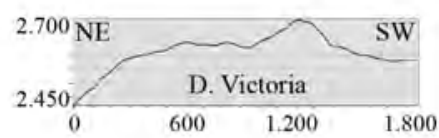

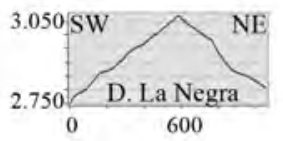

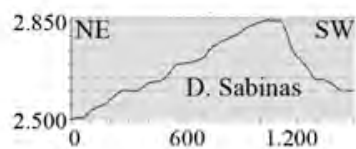
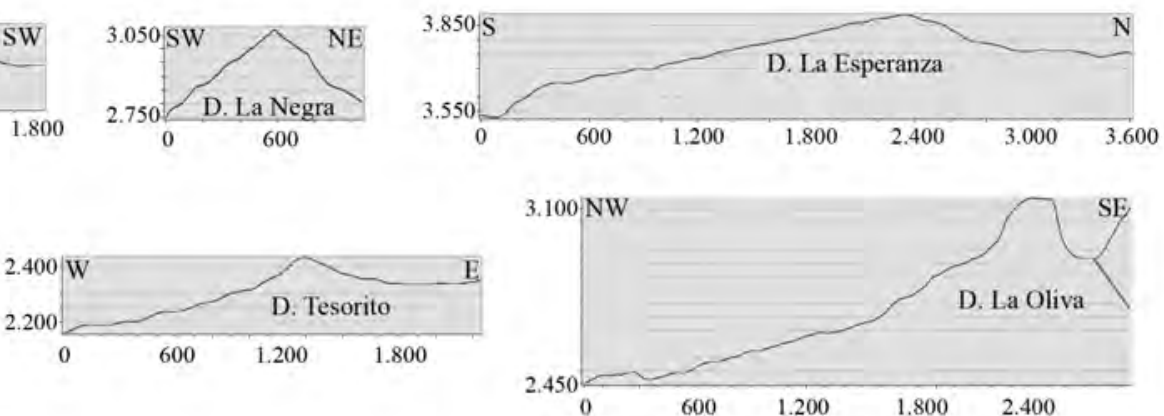

(B)
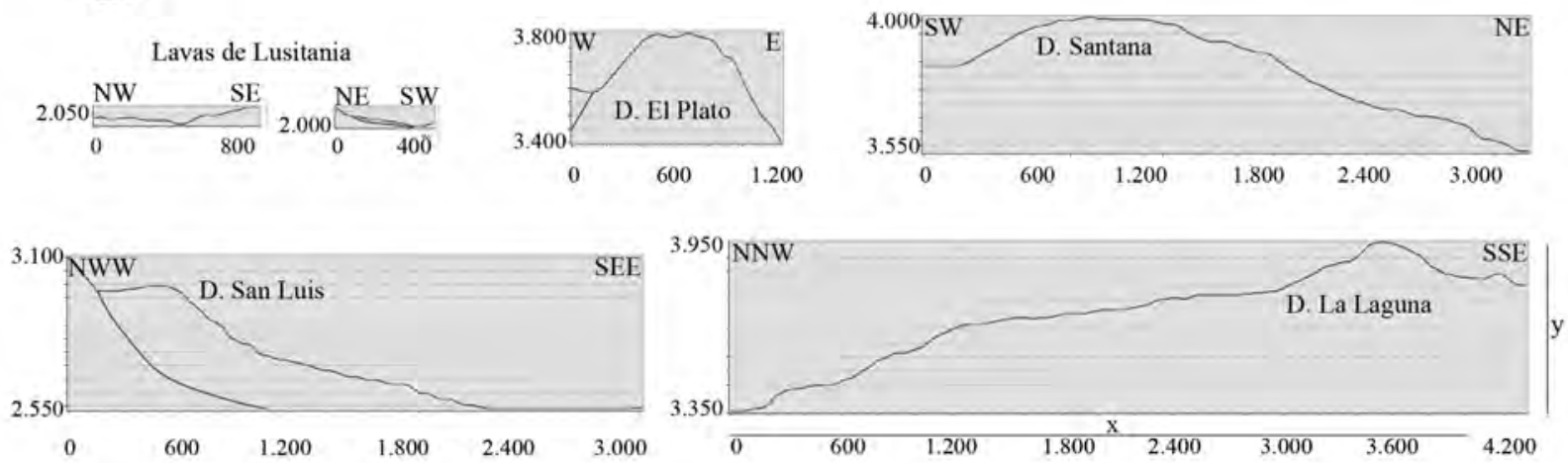

FIGURA 5. Perfiles topográficos (a igual escala) de las expresiones monogenéticas del CVMVT. A. Volcanes monogenéticos "Antiguos" y B. Volcanes monogenéticos "Recientes". Las dimensiones del Domo La Laguna se usan de base para definir la escala; el valor de (x) es de $4 \mathrm{~km}$ y el de (y) es de $600 \mathrm{~m}$, los valores en la vertical corresponden a m.s.n.m.

\section{TEMPORALIDAD}

Los 14 centros volcánicos del CVMVT se pueden subdividir temporalmente en dos grupos según relaciones estratigráficas con productos volcánicos del CVNR. Estos grupos responden a rangos de edad definidos a partir de dataciones y de relaciones estratigráficas, paleoambientales y paleomagnéticas. Así, los volcanes que fueron formados en el intervalo entre 1,8 y 0,045 Ma (Calabriense - Pleistoceno Tardío) se denominan en este trabajo como "Antiguos", mientras aquellos más jóvenes de 0,045 Ma se denominan "Recientes".

Los volcanes "Antiguos", corresponden a los domos Sancancio, Tesorito, Gallinazo, Victoria, Amazonas, La Oliva, Sabinas, La Negra, y La Esperanza; todos asociados al periodo Pre-Ruiz $(1,8 \pm 0,1$ a $0,97 \pm 0,05$ Ma; Martínez et al., 2014); estos volcanes se encuentran suprayacidos por el DA-VT ( $<45.000$ - 35.000 años), con excepción del Domo La Esperanza, que se asocia a este grupo debido a que sus lavas se encuentran afectadas por modelamiento glacial y presentan una relación estratigráfica contemporánea con el Periodo Intermedio Ruiz del CVNR. Se interpreta que este domo fue originado posterior a la erupción que generó la Ignimbrita de Río Claro-Molinos, cuyo depósito tiene una edad de $89 \pm 7$ ka (Schaefer, 1995) (cf. Martínez et al., 2014). Los únicos volcanes monogenéticos con dataciones absolutas en este grupo son los domos Sancancio y Tesorito (K/Ar en roca total de 1,2 $\pm 0,08$ Ma y 1,2 \pm 0,2 Ma, respectivamente; Thouret et al., 1985, 1990) (FIGURA 6). 
Los volcanes “Recientes” (Domos El Plato, La Laguna, Santana, San Luis y Flujo de lava de Lusitania) tienen flujos de lava que se extienden sobre zonas afectadas por modelamiento glacial (i.e. se formaron en los últimos 45.000 años; edad del Máximo Avance Glaciar, Flórez, 1992). Estos volcanes corresponden a aquellos formados posteriormente a la depositación de DA-VT (0,045-0,035 Ma). Según Thouret et al. (1990), durante el periodo eruptivo Ruiz (<0,15 Ma) se emplazaron algunos centros volcánicos monogenéticos, entre estos los domos La Laguna, Santana y El Plato. Este periodo es correlacionable con el Segundo Periodo Eruptivo (<0,045 Ma) de Martínez et al. (2014); tal periodo comprende depósitos con bajo a casi nulo modelado glacial. Finalmente, el Domo San Luis, y el Flujo de lava de Lusitania también pertenecen a este grupo, siendo este último el centro volcánico más joven del campo (<38.000 \pm 16 años, Mejía et al., 2011).

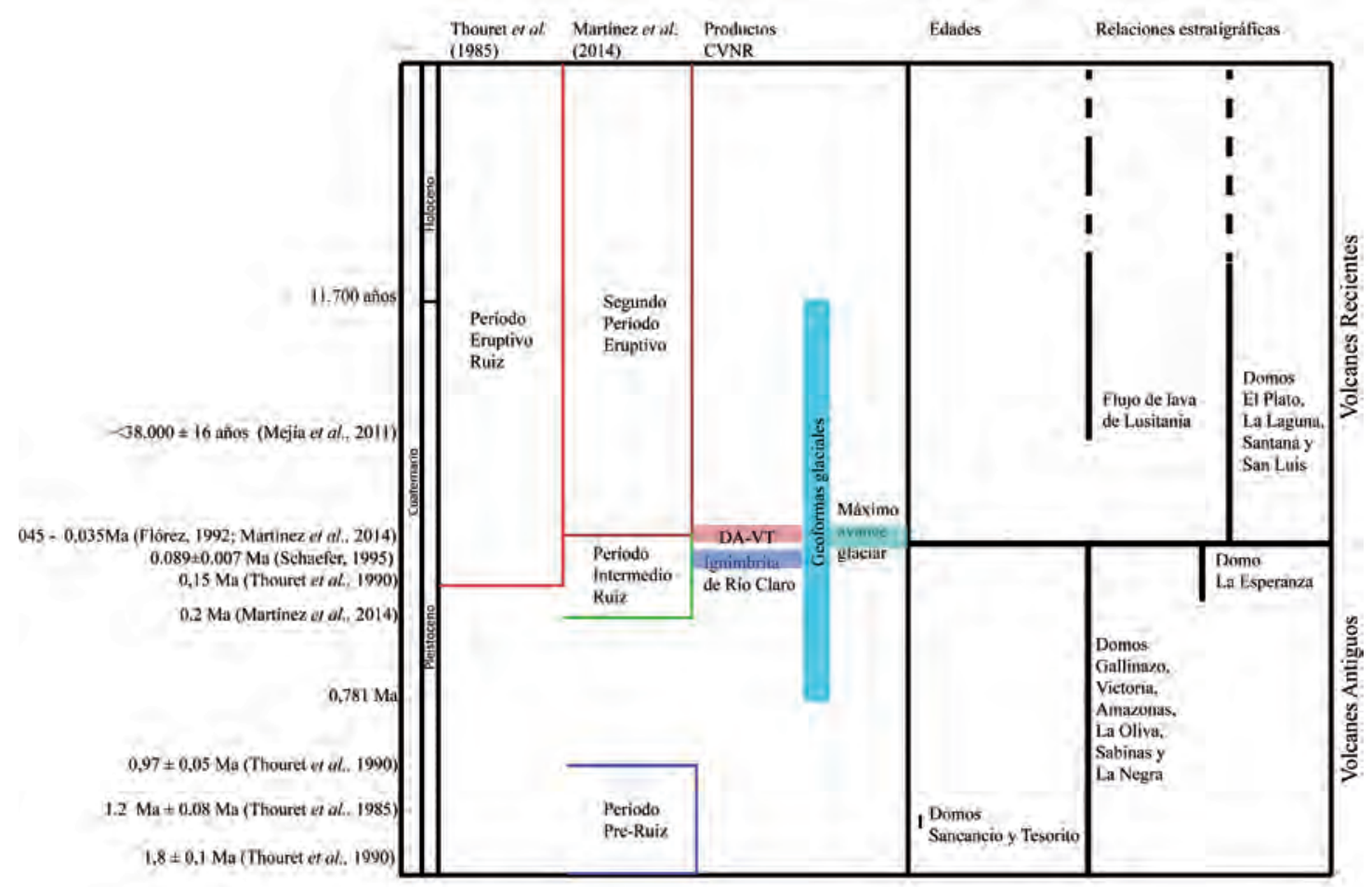

FIGURA 6. Relación temporal de los volcanes pertenecientes al CVMVT. La estratigrafía del CVNR (Thouret et al., 1985; Martínez et al., 2014) y las relaciones paleoambientales fueron tomados como referentes para definir dos temporalidades, marcadas por el inicio del Segundo Periodo Eruptivo del CVNR, el DA-VT y el máximo avance glacial en el sector. Dataciones cuantitativas de algunos volcanes y relaciones estratigráficas, permiten ubicar temporalmente estos volcanes monogenéticos.

\section{GEOMORFOLOGÍA}

Las evidencias morfológicas en el CVMVT, muestran que los volcanes se encuentran muy afectados por la tectónica reciente y procesos de meteorización y erosión ligados con el clima y la vegetación abundante. De esta manera, se han generado por ejemplo, cimas muy pronunciadas relacionadas con los centros de emisión volcánica. A continuación se realiza una descripción de los centros volcánicos con base en su distribución temporal.

\section{Volcanes "Antiguos"}

El Domo Sancancio (FIGURA 7A), con una forma circular, es el volcán ubicado más al NW del CVMVT. Este domo en su flanco SE se encuentra cubierto por depósitos volcaniclásticos compuestos por fragmentos de pómez redondeada de tamaño lapilli medio (en el sentido de Murcia et al., 2013). En la base del domo, se observa una estructura de contracción termal producida por el enfriamiento del magma en superficie ( $c f$. DeGraff y Aydin, 1987; Aydin y DeGraff, 1988) con diaclasas en disposición vertical a sub vertical (FIGURA 7D). En el Domo Tesorito (FIGURA 7B) se puede apreciar también disyunción columnar característica en el flujo de lava asociado (Aristizabal y Echeverri, 2001) (FIGURA 7E), así como depósitos volcaniclásticos suprayacientes sobre su flanco sur que hacen parte del DA-VT (FIGURA 2). El Domo Victoria presenta igualmente disyunción columnar (FIGURA 7F), además de un flujo de lava asociado a su evolución. En el flujo se observa un pico alto, 
producto de erosión asociado a fracturamiento que se encuentra alineado con silletas y hombreras estructurales (FIGURA 7C) relacionados con la falla Samaná Sur (FIGURA 2) y el SFVT. Los domos Tesorito, Amazonas, Sabinas, Gallinazo, La Oliva y Victoria (FIGURA 7G) se encuentran afectados por fallas geológicas locales relacionadas con la falla Samaná Sur en sentido NNESSW y el SFVT con tendencia NWW-SEE (FIGURA 2), lo que genera una meteorización y erosión permanente en sus flancos, dejando cimas pronunciadas características (FIGURA 5A). Los domos La Oliva, Sabinas, La Negra y La Esperanza se emplazan atravesando flujos de lava del volcán Nevado del Ruiz (FIGURA 2) asociadas al Periodo Pre-Ruiz (cf. Martínez et al., 2014). El Domo La
Negra (FIGURA 5A) presenta un flujo de lava asociado, el cual tiene una forma elongada hacia el NW; hacia el SE se encuentra disectado por la falla Pico Terrible con tendencia NNE-SSW, que lo separa de flujos de lava pertenecientes al CVNR (FIGURA 2). El Domo La Esperanza (FIGURA 7H) tiene asociado un flujo de lava hacia el W que suprayace el Stock de Manizales (FIGURA 2). Este domo se encuentra en la intersección de la falla Olleta - Nereidas con Villamaría - Termales (FIGURA 2) y su flujo de lava sigue el trazo del segmento El Ocho del SFVT (FIGURA 3). Este flujo de lava asociado se encuentra rellenando parcialmente valles glaciales antiguos esculpidos en lavas del CVNR en el valle del río Chinchiná (FIGURA 2).

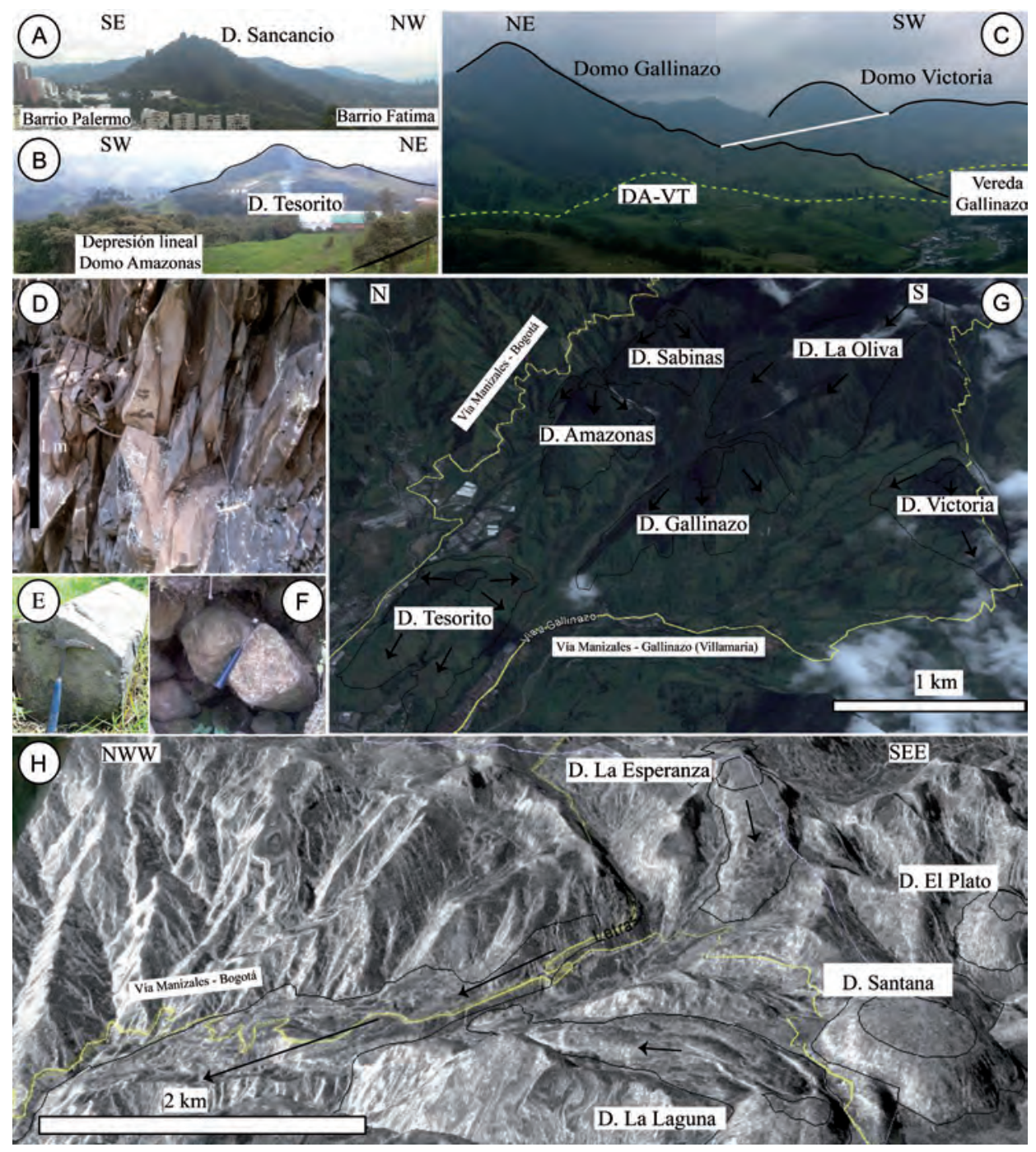

FIGURA 7. Volcanes "Antiguos" del CVMVT. A. Domo Sancancio. B. Depresión lineal sobre la base del Domo Amazonas, en dirección del Domo Tesorito, la cual coincide con un segmento del SFVT. C. Domos Gallinazo y Victoria en la vereda Gallinazo (Villamaría-Caldas); de color blanco lineamiento NNW-SSE. D. Diaclasamiento paralelo debido a la contracción termal, presente en el Domo Sancancio. E. Forma hexagonal producida por el resultado de la contracción termal debido al enfriamiento del magma en superficie (disyunción columnar) presente en el Domo Tesorito. F. Disyunción columnar del Domo Victoria. G. Domos Tesorito, Amazonas, Sabinas, Gallinazo, La Oliva y Victoria (Imagen tomada de Google Earth). H. Domo La Esperanza y su flujo de lava asociado, cerca de los domos "Recientes": La Laguna, Santana y El Plato (Imagen de radar). 


\section{Volcanes "Recientes"}

El Domo La Laguna (FIGURA 8A) se encuentra en la intersección de las fallas Olleta-Nereidas y Villamaría -Termales (FIGURA 2). Su flujo de lava asociado fue encausado en un paleo-valle glacial en dirección hacia el río Chinchiná (FIGURA 2). El Domo Santana (FIGURA 8A) presenta un flujo de lava asociado hacia el NE y se encuentra localmente cubierto por depósitos de caída piroclástica recientes; en algunas de sus paredes presenta disyunción columnar. El Domo El Plato tiene una forma circular un poco elongado hacia el E, y presenta una forma de tazón invertido con una cima semicircular aplanada (FIGURA 8B).
Estos domos intruyen lavas del CVNR (FIGURA 2) asociadas al Periodo Pre-Ruiz (cf. Martínez et al., 2014). El Domo San Luis (FIGURA 8C) se encuentra en la intersección de la falla Santa Rosa con Villamaría -Termales (FIGURA 2). Este domo se caracteriza por presentar un flujo de lava que se emplazó en el valle del río Gualí (FIGURA 2) con una longitud de 2,3 km en dirección E; este flujo de lava presenta una estructura escalonada desde su cima (FIGURA 5B). El Flujo de lava de Lusitania se encuentra directamente sobre el segmento Tesorito del SFVT (FIGURA 3) y la falla San Jerónimo (FIGURA 2); estas lavas presentan una forma irregular e intruyen el DA-VT (FIGURA 2).
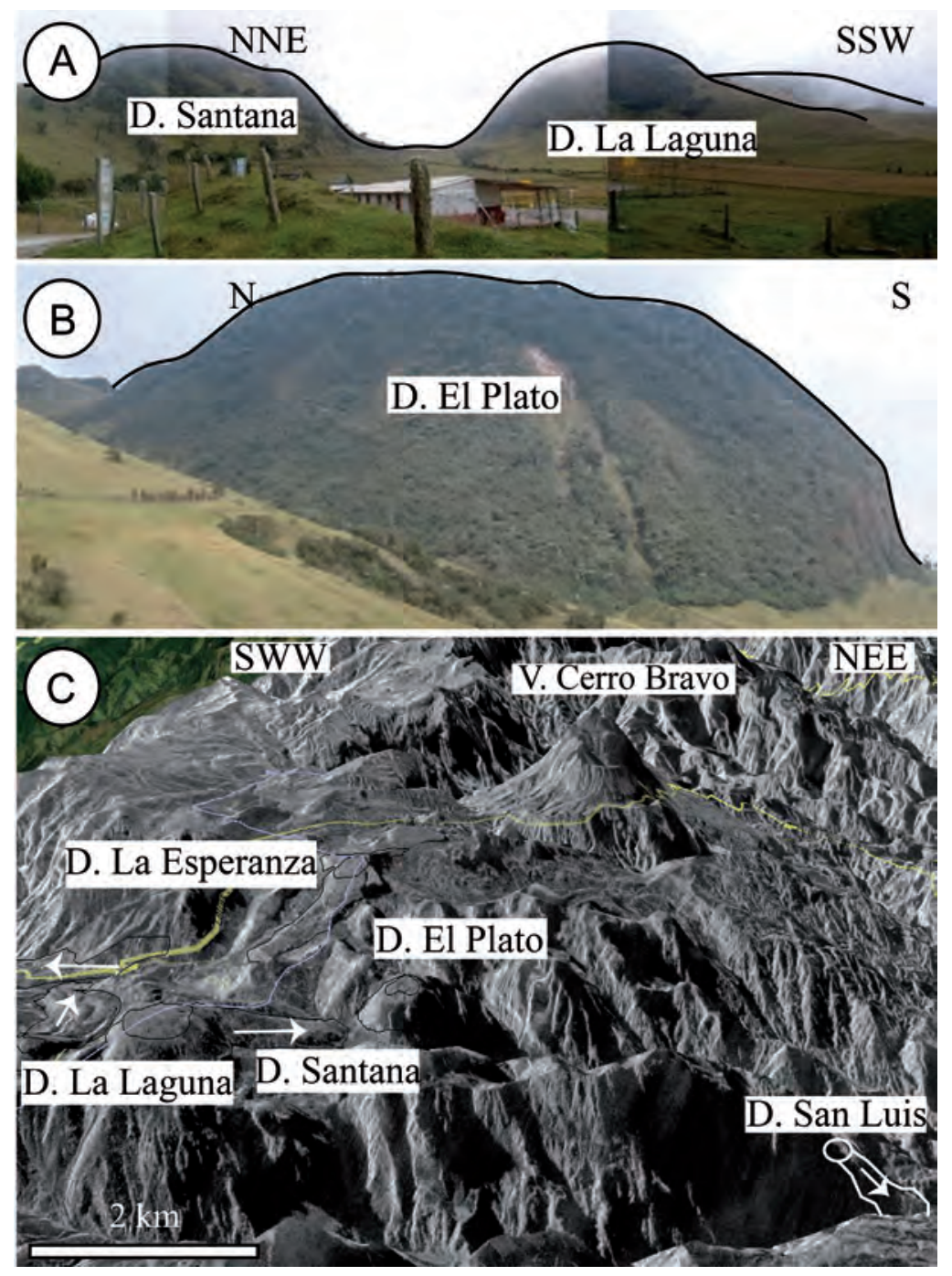

FIGURA 8. Volcanes “Recientes” del CVMVT. A. Domos Santana y La Laguna. B. Domo El Plato, geoforma similar a un “tazón invertido”. C. Domos Recientes La Laguna, Santana, El Plato y San Luis, Domo Antiguo La Esperanza y volcán poligenético Cerro Bravo (Imagen de radar). 


\section{DISCUSIÓN}

\section{Distribución y control estructural en el CVMVT}

Los grupos temporales definidos en el CVMVT, se distribuyen geográficamente respecto al sistema de fallas asociado y a los rangos de temporalidad de los volcanes. Los volcanes "Antiguos" se distribuyen preferencialmente al $\mathrm{W}$ del campo volcánico, sobre los segmentos Sancancio, Tesorito, El Ocho y Termales del SFVT (FIGURA 3), al NW y NNW del CVNR, mientras que los volcanes "Recientes" hacia el centro y E del campo, sobre los segmentos El Ocho y Gualí del SFVT (FIGURA 3). Sin embargo, el Flujo de lava de Lusitania que forma parte de los volcanes "Recientes", se encuentra espacialmente localizado en la parte occidental del CVMVT rodeado de volcanes "Antiguos", evidencia de que no necesariamente existe un patrón en la distribución de los centros volcánicos dentro de un campo (cf. Le Corvec et al., 2013). Al respecto vale la pena mencionar que el centro de emisión del Flujo de lava de Lusitania es desconocido ya que este se encuentra suprayacido por un depósito de avalancha de escombros (cf. Martínez et al., 2014); no obstante, se asume que el origen es cercano al lugar donde aflora.

Los volcanes del CVMVT se encuentran alineados con el SFVT el cual presenta una tendencia NWW-SEE (FIGURA 3), además de una relación con las fallas San Jerónimo, Samaná Sur, Pico terrible, Olleta - Nereidas y Palestina, estas con una tendencia general NNE-SSW en este sector; asimismo afectado por la falla Santa Rosa con tendencia NE-SW, lo que genera zonas fracturadas (FIGURA 9A). El sistema tiene cinemática normalsinistral y es el que posiblemente facilitó el ascenso del magma a la superficie (cf. Borrero et al., 2009).

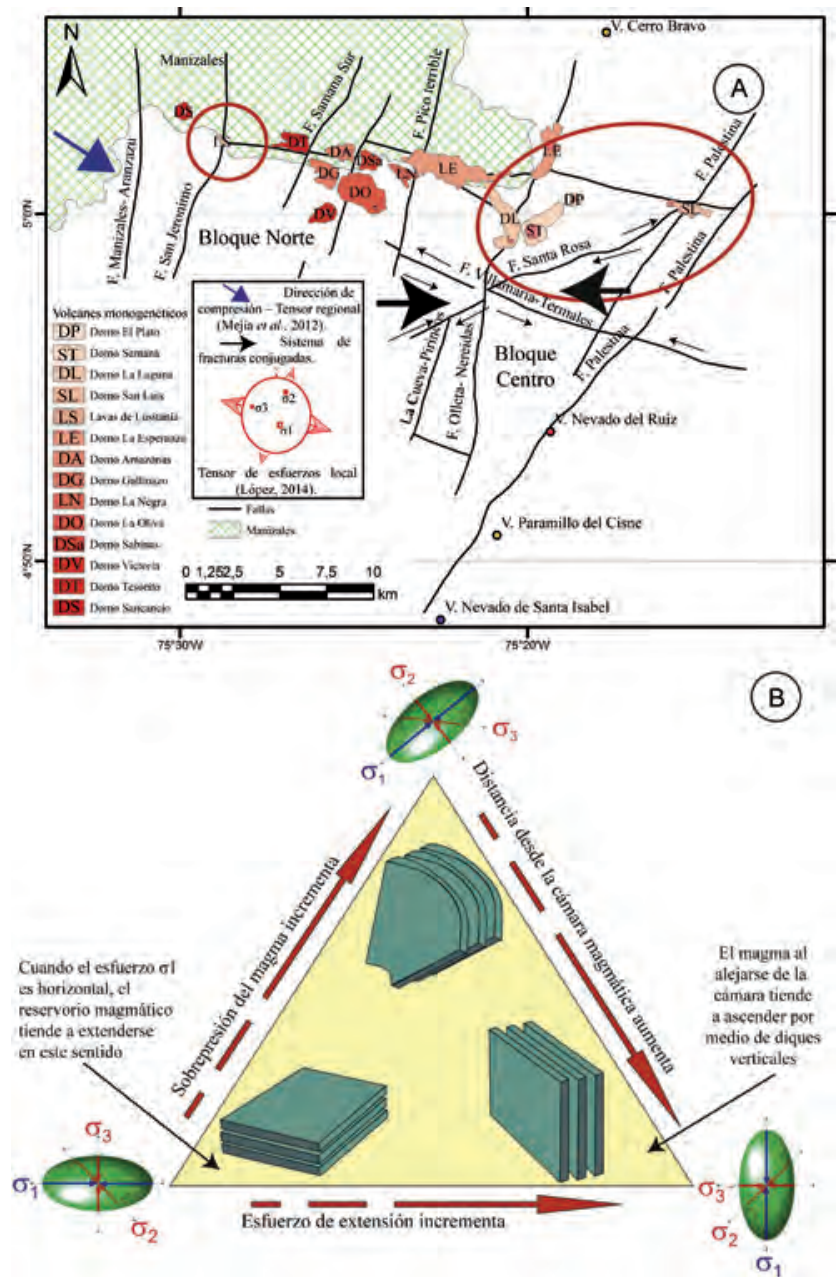

FIGURA 9. A. Ubicación del tensor de esfuerzos regional (Mejía et al., 2012), de $\sigma 1$ entendiendo el sistema de fallas VillamaríaTermales y la falla Santa Rosa como un fracturamiento conjugado y tensor de esfuerzos local para el Bloque Norte (adaptado de López, 2014). Las flechas indican la cinemática asociada. Los óvalos rojos resaltan la ubicación de los volcanes monogenéticos Recientes. B. Relación entre la posición del tensor de esfuerzo con la geometría del sistema de conductos volcánicos (modificado de Tibaldi, 2015), comportamiento propuesto para la fuente magmática asociado al CVMVT. 
Los volcanes "Recientes" se encuentran sobre las fallas en sentido NNE-SSW, las cuales son ortogonales al SFVT, y son consideradas fallas antiguas relacionadas con la falla Palestina. Estas fallas presentan actividad más reciente respecto al SFVT, y son las que lo segmentan. De esta manera, el emplazamiento de los centros volcánicos monogenéticos se encuentra relacionado con estas fallas. Al respecto Martí et al. (2016) menciona que los sistemas de fracturas más recientes son los que tienen la mayor probabilidad de albergar volcanes nuevos. Este hecho sugiere una evolución del campo volcánico en dirección E (FIGURA 9A). La ubicación del Flujo de lava de Lusitania distante de este grupo de domos ubicados al E, sugiere también una tendencia de centros volcánicos nuevos hacia los bordes del campo volcánico con índices de separación y agrupamiento cada vez mayores. Dataciones geocronológicas adicionales (e.g. ${ }^{40} \mathrm{Ar} /{ }^{39} \mathrm{Ar}, \mathrm{U}-\mathrm{Pb},(\mathrm{U}-\mathrm{Th}) / \mathrm{He}$, trazas de fisión) a los volcanes del CVMVT ayudarían a refinar mejor el orden de eventos volcánicos generados, así como la relación existente entre la edad y los procesos de evolución magmática que se dieron en el emplazamiento de los volcanes monogenéticos del CVMVT. Trabajos como el de Leonard et al. (2017) en el campo volcánico de Auckland es un buen ejemplo de la importancia de la geocronología en el entendimiento de la evolución de estos campos.

\section{Modelo de formación del CVMVT}

El CVMVT está conformado por domos cónicos a subcónicos, circulares elongados, con índices de separación y agrupamiento cercanos a cero, especialmente en los volcanes "Antiguos"; esto sugiere mayor disponibilidad de magma y una dinámica estructural favorable para el ascenso hasta la superficie, en un tiempo geológico relacionable con el periodo PreRuiz del CVNR. Estos volcanes monogenéticos muestran un enfriamiento rápido del magma en superficie por sus característicos fracturamientos paralelos y disyunción columnar (FIGURA 7D, E y F); asimismo presentan cimas pronunciadas y flujos de lava asociados.

Con base en las relaciones encontradas, se propone un modelo geológico - estructural basado en el fracturamiento conjugado y presión de fluidos (sobrepresión magmática) para el origen del CVMVT, el cual se encuentra afectado por fallas estructurales con una tendencia NW que sugieren un control tectónico durante su emplazamiento (Borrero et al., 2009).

La falla Santa Rosa con una tendencia NE, al cruzarse con el SFVT, genera un régimen de esfuerzos perpendiculares, donde el esfuerzo $\sigma 1$ (tensor de esfuerzos) se ubica en la bisectriz del ángulo agudo de este cruce de fallas. Este tensor a su vez, presenta diferencias respecto a la dirección de compresión del sistema tectónico colombiano para este sector o tensor regional (actual dirección de compresión NWSE, cf. Mejía et al., 2012). Acorde con Tibaldi (2015), la disposición del esfuerzo $\sigma 1$, el incremento de la sobrepresión, la distancia desde la cámara magmática (fuente magmática) y la influencia tectónica, son patrones que controlan la geometría de los conductos volcánicos; así, en un ambiente tectónico de subducción y a una profundidad en la cual $\sigma 1$ es horizontal, acorde con el tensor regional y el sistema de fracturas conjugadas (FIGURA 9A), la geometría del reservorio magmático se encuentra en disposición horizontal y cambia a medida que la sobrepresión del magma incrementa y que se aleja del lugar de almacenamiento (cámara magmática?) (FIGURA 9B). Este autor también menciona que, al aumentar la distancia desde el fundido magmático hacia la superficie, con un campo de esfuerzos local extensional, la inclinación de los diques alimentadores tiende a ser vertical.

López (2014) propone un modelo sismo-tectónico a partir del cual divide el sector del CVNR en tres zonas (Bloques Norte, Centro y Sur) con base en índices de deformación, mecanismos focales, campos de esfuerzos y estructuras de grandes órdenes como las fallas Palestina, Santa Rosa e Ibagué. El bloque Norte propuesto al norte de la falla Santa Rosa, presenta un carácter distensivo marcado, evidenciado por la presencia de volcanes monogenéticos y patrones de erosión importantes; asimismo en este bloque, se presentan diferencias marcadas en los ejes $\sigma 1$ y $\sigma 3$ en comparación con el bloque regional del sistema tectónico colombiano, donde $\sigma 1$ se encuentra en sentido vertical (en el contexto local). Este sistema de esfuerzos, además de la presión de poros, la cual disminuye la resistencia del material facilitando su ruptura, permite el ascenso de diques verticales (FIGURA 9A). Con base en esto, se visualiza un proceso de ascenso rápido del magma facilitado por diques que intruyen rocas afectadas por fallas estructurales, teniendo presente que los volcanes monogenéticos se encuentran emplazados sobre o muy cerca del SFVT (FIGURA 3 y TABLA 1).

Para el CVMVT, las posibles fuentes de magma estarían a $4 \mathrm{~km}$ de profundidad respecto al nivel del mar (Londoño y Sudo, 2003; Stix et al., 2003; GonzálezGarcia et al., 2015) o a un reservorio magmático localizado a $20-30 \mathrm{~km}$ de profundidad (Londoño, 
2016; Murcia et al., en prensa). Considerando la fuente de $4 \mathrm{~km}$ como la que alimenta el vulcanismo del CVMVT, es posible sugerir que éste está asociado a la liberación de la sobrepresión adquirida por la recarga de magma desde el reservorio más profundo $(20-30 \mathrm{~km})$. Al disminuir la sobrepresión debido al ascenso magmático y conforme el fundido se aleja del reservorio, incluyendo un transporte a través del SFVT, se genera una serie de diques cada vez más verticales que aprovechan los espacios dilatantes de la rocas foliadas del basamento, compuesto de rocas metamórficas del Complejo Cajamarca y rocas miloníticas generadas por metamorfismo (regional y dinámico) que afecta las rocas sedimentarias del Complejo Quebradagrande, el Stock de Manizales y las lavas del CVNR. Esta serie de diques magmáticos están controlados y ascienden por medio de esta foliación, fracturamiento y fallamiento profundo, en este caso para llegar a la superficie como manifestaciones de vulcanismo monogenético.

\section{CONCLUSIONES}

Al noroeste del CVNR se encuentra ubicado el CVMVT, un campo volcánico compuesto de 12 centros volcánicos cónicos y subcónicos (domos), un macizo (Domo La Esperanza) y uno irregular (Flujo de lava de Lusitania) en un área de $114,5 \mathrm{~km}^{2}$. Este campo se encuentra asociado temporalmente al magmatismo que alimenta el CVNR.

El CVMVT se subdividide en volcanes "Antiguos" los cuales se relacionan directamente con el trazo del sistema de fallas Villamaría-Termales, y volcanes "Recientes" los cuales están asociadas con el cruce ortogonal de fallas con tendencia NNE-SSW y los segmentos de dicho sistema.

Los índices de agrupamiento, separación y el rango temporal de los centros volcánicos se relacionan con una tendencia de formación de nuevos volcanes hacia los límites externos del campo volcánico. Esto permite sugerir que existe mayor probabilidad de ocurrencia de un nuevo volcán monogenético hacia los límites externos del CVMVT. Lo anterior está ligado con otros factores como aporte magmático en profundidad y en superficie, cruces de fallas ortogonales entre sí y espacios dilatantes generados por la dinámica estructural de la zona que podrían servir de zonas de debilidad para nuevos ascensos de magma.

\section{AGRADECIMIENTOS}

Este trabajo fue desarrollado en el Instituto de Investigaciones en Estratigrafía - IIES (Universidad de Caldas); agradecemos a todos los integrantes por su apoyo y uso de materiales e instalaciones. Así mismo, agradecemos a Martha Gabriela Gómez Vasconcelos y John J. Sánchez por sus valiosos comentarios y recomendaciones en la revisión de este trabajo.

\section{REFERENCIAS}

Aristizabal, M., y Echeverry, L. (2001). Volcán Tesorito, geología detallada a escala 1:2.000 y modelo evolutivo. Tesis, Facultad de Ciencias Exactas y Naturales, Programa de Geología, Universidad de Caldas, Manizales, Colombia.

Ancochea, E., Naranjo, J.L., Fuster, J.M., y Borrero, C. (1991). Geoquímica de las lavas antiguas del Volcán del Ruiz (Colombia), Sector Noroccidental. Simposio sobre Magmatismo Andino y su Marco Tectónico. Manizales, Colombia. Memorias Tomo I: 65-79.

Ayala, L. (2009). Petrografía y modelo vulcanológico del volcán Nevado del Ruiz, etapa ancestral. Tesis. Facultad de Ciencias Exactas y Naturales, Programa de Geología, Universidad de Caldas, Manizales, Colombia.

Aydin, A., and DeGraff, J.M. (1988). Evoluton of polygonal fracture patterns in lava flows. Science, 239(4839), 471-476. doi: 10.1126/ science.239.4839.471.

Borrero, C., Toro, L.M., Alvarán, M., and Castillo, H. (2009). Geochemistry and tectonic controls of the effusive activity related with the ancestral Nevado del Ruiz volcano, Colombia. Geofísica Internacional, 48(1), 149-169.

Cañón-Tapia, E. (2016). Reappraisal of the significance of volcanic fields. Journal of Volcanology and Geothermal Research, 310, 26-38. doi: 10.1016/j. jvolgeores.2015.11.010.

Connor, C.B., and Conway, F.M. (2000). Basaltic volcanic fields. In: H. Sigurdsson, B. Houghton, 
S.R. McNutt, H. Rymer, and J. Stix (Ed.). The encyclopedia of volcanoes (pp. 423-439). 2nd edition. San Diego: Academic Press, Elsevier.

Cuellar, M., Sanchez, C., y Valencia, M. (2003). Caracterización petrográfica y deformativa de las rocas aflorantes en los alrededores de la falla San Jerónimo, al este del municipio de Manizales. Tesis, Facultad de Ciencias Exactas y Naturales, Programa de Geología, Universidad de Caldas, Manizales, Colombia.

DeGraff, J.M., and Aydin, A. (1987). Surface morphology of columnar joints and its significance to mechanics and direction of joint growth. Geological Society of America Bulletin, 99, 605-617.

De Silva, S., and Lindsay, J.M. (2015). Primary volcanic landforms. In: H. Sigurdsson, B. Houghton, S.R. McNutt, H. Rymer, and J. Stix (Ed.). The encyclopedia of volcanoes (pp. 273-297). 2nd edition. San Diego: Academic Press, Elsevier. doi: 10.1016/B978-0-12-385938-9.00015-8.

Favalli, M., Karátson D., Mazzarini, F., Pareschi, M.T., and Boschi, E. (2009). Morphometry of scoria cones located on a volcano flank: A case study from Mt. Etna (Italy) based on highresolution LiDAR data. Journal of Volcanology and Geothermal Research, 186(3-4), 320-330. doi: 10.1016/j.jvolgeores.2009.07.011.

Flórez, A. (1992). Los Nevados de Colombia glaciales y glaciaciones. Bogotá: Instituto Geográfico Agustín Codazzi.

Gómez, J., Montes, N.E., Novia, A., y Diederix, H. (2015). Mapa Geológico de Colombia (2015). Escala, 1, 1000000. Servicio Geológico Colombiano.

González-Garcia, J., Hauser, J., Annetts, D., Franco, J., Vallejo, E., and Regenauer-Lieb, K. (2015). Nevado Del Ruiz Volcano (Colombia): a 3D model combining geological and geophysical information. World Geothermal Congress. Melbourne, Australia.

González-Garcia, J., and Jessell, M. (2016). A 3D geological model for the Ruiz-Tolima Volcanic
Massif (Colombia): Assessment of geological uncertainty using a stochastic approach based on Bézier curve design. Tectonophysics, 687, 139157. doi: 10.1016/j.tecto.2016.09.011.

González, L., y Jaramillo, C.M. (2002). Estudio neotectónico multidisciplinario aplicado a la Falla Villamaría Termales. Tesis, Facultad de Ciencias Exactas y Naturales, Programa de Geología, Universidad de Caldas, Manizales.

Grosse, P., van Wyk de Vries, B., Petrinovic, I.A., Euillades, P.A., and Alvarado, G.E. (2009). Morphometry and evolution of arc volcanoes. Geology, 37(7), 651-654. doi: 10.1130/ G25734A.1.

Karátson, D., Favalli, M., Tarquini, S., Fornaciai, A., and Wörner, G. (2010). The regular shape of stratovolcanoes: A DEM-based morphometrical approach. Journal of Volcanology and Geothermal Research, 193(3-4), 171-181. doi: 10.1016/j. jvolgeores.2010.03.012.

Kereszturi, G., and Németh, K. (2012). Monogenetic basaltic volcanoes: Genetic classification, growth, geomorphology and degradation. In: K. Németh (Ed.). Updates in volcanology - new advances in understanding volcanic systems (pp 3-88). Auckland: InTech. doi: 10.5772/51387.

Leonard, G.S., Calvert, A.T., Hopkins, J.L., Wilson, C.J., Smid, E.R., Lindsay, J.M., and Champion, D.E. (2017). High-precision ${ }^{40} \mathrm{Ar} /{ }^{39} \mathrm{Ar}$ dating of quaternary basalts from Auckland volcanic field, New Zealand, with implications for eruption rates and paleomagnetic correlations. Journal of Volcanology and Geothermal Research, 343, 6074. doi: 10.1016/j.jvolgeores.2017.05.033.

Le Corvec, N., Spörli, K.B., Rowland, J., and Lindsay, J. (2013). Spatial distribution and alignments of volcanic centers: clues to the formation of monogenetic volcanic fields. EarthScience Reviews, 124, 96-114. doi: 10.1016/j. earscirev.2013.05.005.

Londoño, J.M. (2016). Evidence of recent deep magmatic activity at Cerro Bravo-Cerro Machín volcanic complex, central Colombia. Implications for future volcanic activity at Nevado del Ruiz, 
Cerro Machín and other volcanoes. Journal of Volcanology and Geothermal Research, 324, 156168. doi: 10.1016/j.jvolgeores.2016.06.003.

Londoño, J.M., and Sudo, Y. (2003). Velocity structure and a seismic model for Nevado del Ruiz Volcano (Colombia). Journal of Volcanology and Geothermal Research, 119(1), 61-87. doi: 10.1016/S0377-0273(02)00306-2.

López, C. (2014). Modelo sismo-tectónico de los principales sistemas de fallas en el sector del volcán nevado del Ruiz y su relación con el sistema volcánico. Tesis MSc, Universidad de Caldas, Colombia.

Martínez, L., Valencia L., Ceballos, J., Narváez, B., Pulgarín, B., Correa, A., Navarro, S., Murcia, H., Zuluaga, I., Rueda, J., y Pardo, N. (2014). Geología y estratigrafía del Complejo Volcánico Nevado del Ruiz. Informe final, Bogotá - Manizales Popayán. Servicio Geológico Colombiano. 853p.

Marti, J., López, C., Bartolini, S., Becerril, L., and Geyer, A. (2016). Stress controls of monogenetic volcanism: a review. Frontiers in Earth Science, 4, 106, 1-17. doi: 10.3389/feart.2016.00106.

Mejía, V., Sánchez-Duque, A., Opdyke, N., Huang, K., Rosales, A., and Agudelo, C. (2011). VolcanoTectonic implications for the Ruiz-Tolima Volcanic Complex based on paleomagnetic data. Biennial Meeting of LATINMAG (Latin American Association of Paleomagnetism and Geomagnetism), Tandil, Argentina.

Mejía, E.L., Velandia, F., Zuluaga, C.A., López, J.A., y Cramer, T. (2012). Análisis estructural al noreste del volcán Nevado del Ruíz, Colombia-aporte a la exploración geotérmica. Boletín de Geología, 34(1), 27-41.

Miallier, D., Pilleyre, T., Boivin, P., Labazuy, P., Gailler, L.S., and Rico, J. (2017). Grand Sarcoui volcano (Chaîne des Puys, Massif Central, France), a case study for monogenetic trachytic lava domes. Journal of Volcanology and Geothermal Research, 345, 125-141. doi: 10.1016/j.jvolgeores.2017.07.015.

Montoya, A., y Torres, A.H. (2005). Cartografía, análisis metalográfico y petrográfico de los pórfidos ubicados al este del municipio de Manizales, sector de Gallinazo. Tesis, Facultad de Ciencias Exactas y Naturales, Programa de Geología, Universidad de Caldas, Manizales, Colombia.

Murcia, H.F., Borrero, C.A., Pardo, N., Alvarado, G.E., Arnosio, M., y Scolamacchia, T. (2013). Depósitos volcaniclásticos: Términos y conceptos para una clasificación en español. Revista Geológica de América Central, 48, 15-39.

Murcia, H., Borrero, C., and Németh, K. (in press). Overview and plumbing system implications of monogenetic volcanism in the northernmost Andes' volcanic province. Journal of Volcanology and Geothermal Research. doi: 10.1016/j. jvolgeores.2018.06.013.

Naranjo, J.L., y Ríos, P.A. (1989). Geología de Manizales y sus alrededores y su influencia en los riesgos geológicos. Manizales: Universidad de Caldas, 10(1-3). 112p.

Németh, K. (2010). Monogenetic volcanic fields: Origin, sedimentary record, and relationship with polygenetic volcanism. In: E. Cañón-Tapia, and A. Szakács (Ed.). What is a volcano? (pp. 43-66). Boulder: Geological Society of America. Vol. 470. doi: 10.1130/2010.2470(04).

Porter, S. (1972). Distribution, morphology and size frequency of cinder cones on Mauna Kea volcano, Hawaii. Geological Society of America Bulletin, 83(12), 3607-3612.

Rayo, L. (2012). Evolución geoquímica y térmica del volcán Nevado del Ruiz, Colombia. M.Sc. Tesis, Universidad Nacional de Colombia, Bogotá, Colombia.

Samacá-Torres, W. (2016). Análisis morfométrico y Geomorfológico de la Caldera de Paletará (Cauca), Colombia. Tesis M.Sc., Universidad Nacional de Colombia, Bogotá, Colombia.

Schaefer, S.J. (1995). Nevado del Ruiz volcano, Colombia: magmatic system and evolution. Ph.D. Thesis, Arizona State University, USA.

Settle, M. (1979). The structure and emplacement of cinder cone fields. American Journal of Science, 279, 1089-1107. doi: 10.2475/ajs.279.10.1089. 
Shane, P., and Coote, A. (2018). Thermobarometry of Whangarei volcanic field lavas, New Zealand: Constraints on plumbing systems of small monogenetic basalt volcanoes. Journal of Volcanology and Geothermal Research, 354, 130139. doi: 10.1016/j.jvolgeores.2018.02.013.

Stix, J., Layne, G.D., and Williams, S.N. (2003). Mechanisms of degassing at Nevado del Ruiz volcano, Colombia. Journal of the Geological Society, 160(4), 507-521.

Thouret, J.C., Cantagrel, J.M., Salinas, R., and Murcia, A. (1990). Quaternary eruptive history of Nevado del Ruiz (Colombia). Journal of Volcanology and Geothermal Research, 41(1-4), 225-251. doi: 10.1016/0377-0273(90)90090-3.

Thouret, J.C., Vatin-Perignon, N., Cantagrel, J.M., Salinas, R., and Murcia, A. (1985). Le Nevado el Ruiz (Cordillère Centrale des Andes de Colombie): Stratigraphie, structures et dynamisme d'un appareil volcanique andésitique, compose et polygénique. Revue de Geographie Physique et de Geologie Dynamique, 26, 257-271.

Tibaldi, A. (2015). Structure of volcano plumbing systems: A review of multi-parametric effects. Journal of Volcanology and Geothermal Research, 298, 85-135.

Toro, L.M., Alvarán-Echeverri, M., y Borrero-Peña, C.A. (2008). Rocas con afinidad adakítica al sur-este de Manizales: rasgos petrogenéticos y geoquímicos. Boletín de Geología, 30(2), 49-60.

Villagómez, D., and Spikings, R. (2013). Thermochronology and tectonics of the Central and Western Cordilleras of Colombia: Early
Cretaceous-Tertiary evolution of the Northern Andes. Lithos, 160-161, 228-249. doi: 10.1016/j. lithos.2012.12.008.

Villagómez, D., Spikings, R., Magna, T., Kammer, A., Winkler, W., and Beltrán, A. (2011). Geochronology, geochemistry and tectonic evolution of the Western and Central Cordilleras of Colombia. Lithos, 125(3-4), 875-896. doi: 10.1016/j.lithos.2011.05.003.

Wood, C.A. (1980). Morphometric evolution of cinder cones. Journal of Volcanology and Geothermal Research, 7(3-4), 387-413. doi: 10.1016/03770273(80)90040-2.

\begin{tabular}{c}
\hline Luis Alvaro Botero-Gómez \\
ORCID: 0000-0002-0783-4097 \\
Pablo Osorio \\
ORCID: 0000-0001-7523-9137 \\
Hugo Murcia \\
ORCID: 0000-0002-3570-1988 \\
Carlos Borrero \\
ORCID: 0000-0002-4907-2078 \\
Jeny Alejandra Grajales \\
ORCID: 0000-0002-6966-9971 \\
\hline \hline
\end{tabular}

Trabajo recibido: febrero 14 de 2018

Trabajo aceptado: mayo 26 de 2018 